\title{
Robustness of Focused and Global Impedance Estimates of Bladder Volumes against Uncertainty of Urine Conductivity
}

\author{
Víctor Hugo Mosquera Leyton ${ }^{1}$, Ramon Bragós Bardia ${ }^{2}$ and \\ Carlos Felipe Rengifo Rodas ${ }^{1}$ \\ ${ }^{1}$ Department of Electronic, instrumentation and control, Universidad del Cauca, \\ Popayán, Colombia \\ ${ }^{2}$ Department of Electronic Engineering, Technical University of Catalonia, \\ Barcelona, Spain \\ E-mail: mosquera@unicauca.edu.co \\ January 2020
}

\begin{abstract}
Bioimpedance measurements are currently used to monitor various biological processes and are potentially useful for studies of urodynamics. Global impedance (GI) and focused impedance measurements (FIM) can be used to monitor bladder volumes, but these are subject to varying conductivity of urine. To address this, we emulated a human bladder using an agar phantom filled with saline solutions of varying conductivities and estimated volumes using a modified FIM-based approach. Using this novel strategy, electrical potentials did not change significantly with constant liquid volumes, even when the conductivity of the saline solutions was varied between 1.027 to 1.877 and $2.610 \mathrm{~S} / \mathrm{m}$. Conversely, GI and classic FIM measurements of constant liquid volumes varied with conductivity. These observations suggest that the proposed FIM approach is suitable for bladder volume estimation due to its robustness against uncertainties of conductivity. The bioimpedance hardware used in our experiments comprised 8 electrodes and a a small and low cost impedance measurement system based on an AFE4300 direct impedance measurement device.
\end{abstract}

Keywords: Bioimpedance, focused impedance measurement, bladder emptying, urine monitor.

\section{Introduction}

Urinary incontinence (UI), detrusor overactivity and prostatic hyperplasia are pathologies that cause urinary tract infections due to loss of sphincter control. UI is a common condition in elderly people, and between $15 \%$ to $35 \%$ of all adults suffer from severe UI $[1,2]$. Additionally, people suffering from spinal cord injuries are highly prone to urinary tract infection due to the loss of micturition sensation. According to 
the World Health Organization, the prevalence of urinary tract diseases ranges from 40 to 80 per million. Hence, UI is a public health problem [3, 4, 5]. UI affects selfesteem and quality of life, and is associated with higher rates of depression. UI also affects daily activities such as work, travel, social interaction, physical activity, sexual function, and sleep [6, 7]. Currently, catheterization is the most commonly used method for evacuating the bladder for people with UI, but the periodicity of the associated emptying procedures depends on an empirical estimate of the time it takes to fill the patient's bladder. Underestimating this period unnecessarily increases the number of catheterization procedures, thus increasing the risk of infections, cancers, and kidney stones [8, 9]. Conversely, overestimation of the catheterization period increases the risk of complications such as over-distension of the bladder wall, hydronephrosis, and autonomic dysreflexia [8]. Ultrasound-based estimates are the gold standard measure of bladder volumes, with advantages of high portability and accuracy. However, ultrasound measurements must be performed by professionals, precluding continuous monitoring and limiting application to bladder volume monitoring [10].

Due to the electrical conductivity of the urine, several bioimpedance techniques have been considered for bladder volume monitoring [11]. Among these, Global Impedance (GI) is too sensitive to changes in the conductivity of the urine [8, 12]. Similarly, average conductivity index measurements [13] are subject to urine conductivity.

Another bioimpedance approach that is applied to bladder volume monitoring is based in voltage change ratios (VCR) [10]. In a previous study using VCR [10], electrode locations of a four-electrode system were optimized, but the conductivity of the urine was not taken into consideration. Similarly to VCR, the focused impedance method (FIM) [14, 15] has been successfully used to estimate organ volumes in simulations [16, 17] and for live monitoring of stomach emptying [18] and pulmonary ventilation [19]. Despite the promise of FIM, no studies demonstrate the robustness of the ensuing volume estimates to changes in the conductivity of fluids.

To our knowledge, the most promising bladder monitoring data were produced using the Impedance Ratio Method (IRM) [12], for which tests on a phantom showed independence of estimated volumes on conductivity of saline solutions. In this paper, we propose a modification of the FIM approach that produces similar results to those from IRM. Additionally, we compare bladder volume estimates from GI and FIM under controlled conditions of saline solutions with varying conductivities. Robustness against uncertainties in conductivity is essential for bladder bladder volume estimates, because the conductivity of urine varies with health status and diet [20].

\section{Materials and Methods}

\subsection{Bladder phantom}

The bladder phantom was constructed using $20 \mathrm{~g}$ of biological agar No, 1 of (Oxoid ${ }^{\mathrm{TM}}$ ) in $1000-\mathrm{cm}^{3}$ saline solutions in a methacrylate cubic containers of $30 \mathrm{~cm}$ in length, 
width and height. This container was filled with $4.5 \mathrm{~cm}$ in height of agar. These 1L saline/agar solutions had a conductivity of $0.193 \mathrm{~S} / \mathrm{m}$. The overall conductivity of the phantom was set to $0.217 \mathrm{~S} / \mathrm{m}$ to emulate the electrical characteristics of the lower pelvis in humans $[20,21]$. This conductivity value was verified using measurements with a Hewlett Packard 4192A impedance analyzer. In these experiments, three phantoms with 1.9-cm deep cylindrical cavities and radii of $5.2,4.15$ and $2.5 \mathrm{~cm}$ were used to emulate bladders of different sizes. The 8 electrodes of the EIT system were located on the underside of the container, according to the pattern shown in Figure 1 . Saline solutions in these cavities had conductivity values of $1.027,1.871$ and $2.610 \mathrm{~S} / \mathrm{m}$, as described in [13] and [21]. Because the conductivity of the bladder wall is similar to that of the organs of the lower pelvis, it was not necessary to model this tissue [20].

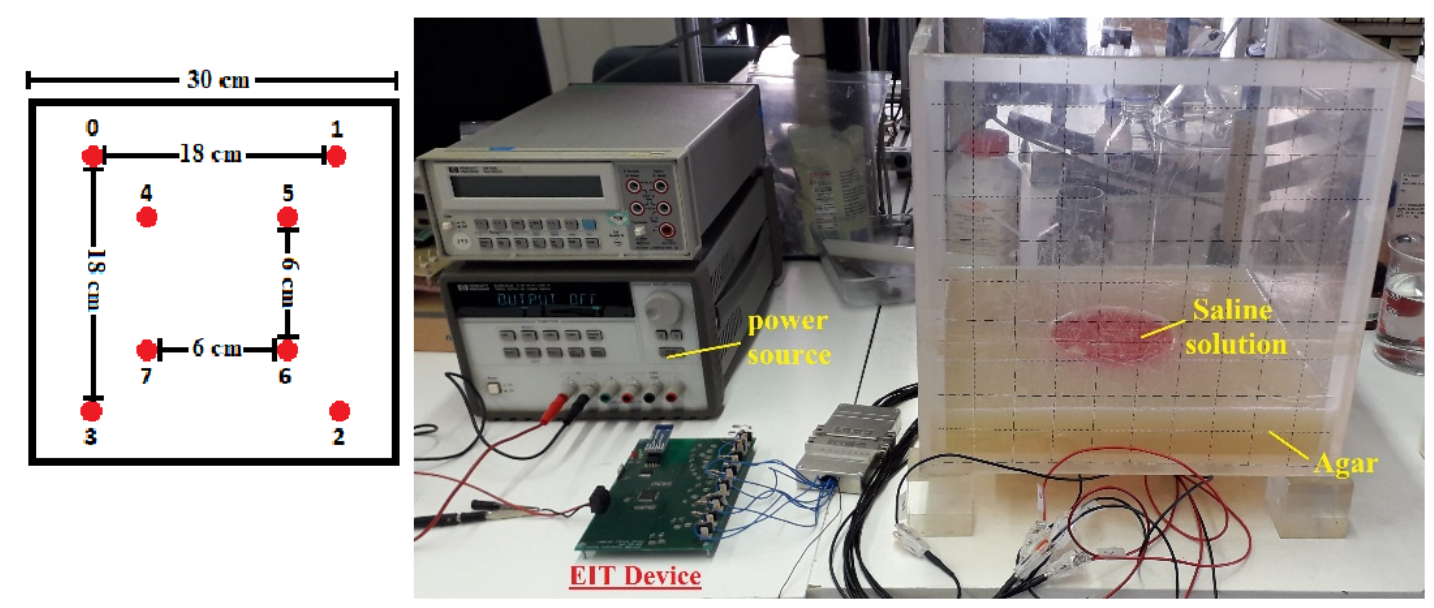

Figure 1. Electrodes configuration and experiment assembly. The red dots of the top image represent the position of the electrodes in the underside of the container.

\subsection{EIT system}

A low-cost EIT system for bladder monitoring was proposed previously [22]. This device comprises an analog front-end AFE4300 [23] instrument with 8 electrodes for current injection and voltage measurements. Current is applied with a peak-to-peak amplitude of $833 \mu \mathrm{A}$ and a frequency of $50 \mathrm{kHz}$, and sinusoidal signals are generated using a digital direct synthesizer coupled with a voltage controlled current source. The analog multiplexers are configured through registers to ensure adjacent patterns for injections and measurements. The internal circuit of the AFE4300 performs a full wave rectification of the voltage signal measured by the electrodes, and delivers this value as a 16-bit word to the PIC 16F887 microcontroller instrument. The communication protocol between the AFE4300 and the PIC 16F887 is the Serial Peripheral Interface (SPI). The microcontroller is also used to configure the registers of the AFE4300 and to send voltage reads via Bluetooth to a Labview application that stores the frames.

Figure 1 shows the experimental assembly. 


\subsection{Focused Impedance Measurements}

Eight-electrode tetrapolar FIMs are based on the sum of independent measurements of mutually perpendicular and concentric potentials, which allow detection of changes that are generated by the injected current on the equipotential lines inside the object under study. Saha and collaborators [24] show that changes in conductivity/impedance of the object under study lead to proportional differences in electrical potential between equipotential lines. This assumption remains valid for a constant injected current.

The arrangement of electrodes for FIM is shown in Figure 2. When the current is injected through the $E_{0}$ and $E_{2}$ electrodes, equipotential lines are represented as $a-a^{\prime}$ and $b-b^{\prime}$. Similarly, when current is injected through the $E_{1}$ and $E_{3}$ electrodes, then the equipotential lines are represented as $c-c^{\prime}$ and $d-d^{\prime}$. These potentials are measured on electrodes $E_{4}, E_{5}, E_{6}$ and $E_{7}$, and the focused area $\mathbf{O}$ is defined for equipotential lines $[17]$.

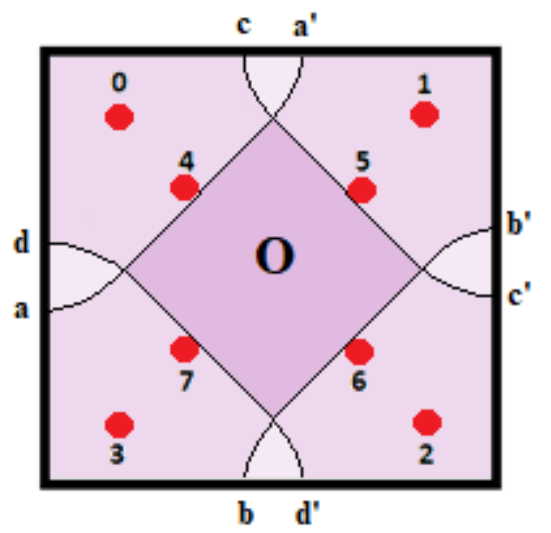

Figure 2. Focused area in an 8-Electrodes FIM arrangement.

Changes in potential are estimated relative to the reference measure $P_{\text {ref }}$, which is the potential when there is no object in the region $\mathbf{O}$ of Figure 2. In this case, the conductivity is homogeneous throughout the container. $P_{\text {ref }}$ is calculated using equation 1 as follows:

$$
P_{\text {ref }}=P_{4,6}^{o}+P_{5,7}^{o}
$$

$P_{4,6}^{o}$ is the difference in potential between the electrodes $E_{4}$ and $E_{6}$ for the homogeneous configuration (indicated with the superscript ${ }^{o}$ ). Similarly, $P_{5,7}^{o}$ is the difference in potential between the electrodes $E_{5}$ and $E_{7}$. Hence, given a conductivity change in region $\mathbf{O}$ (Figure 2), the potential is defined in equation 2 as follows:

$$
P=P_{4,6}+P_{5,7}
$$

The variability of potential is then defined as:

$$
\Delta P=\frac{P-P_{r e f}}{P_{r e f}}
$$


Table 1. The proposed FIM approaches, unlike the classical FIM, use adjacent patterns for injection and measurement

\begin{tabular}{|c|c|c|c|}
\cline { 2 - 4 } \multicolumn{1}{c|}{} & Injection & \multicolumn{2}{c|}{ Measurement } \\
\hline \multirow{4}{*}{$\begin{array}{c}\text { Potentials on } \\
\text { internal electrodes }\end{array}$} & $E_{0}-E_{1}$ & $P_{4,5}$ & $P_{5,6}$ \\
\cline { 2 - 4 } & $E_{1}-E_{2}$ & $P_{5,6}^{\prime}$ & $P_{6,7}$ \\
\cline { 2 - 4 } & $E_{4}-E_{5}$ & \multicolumn{2}{|c|}{$P_{6,7}$} \\
\cline { 2 - 4 } & $E_{5}-E_{6}$ & \multicolumn{2}{|c|}{$P_{4,7}$} \\
\hline \multirow{2}{*}{$\begin{array}{c}\text { Potentials on } \\
\text { external electrodes }\end{array}$} & $E_{4}-E_{5}$ & $P_{0,1}$ & $P_{1,2}$ \\
\cline { 2 - 4 } & $E_{5}-E_{6}$ & $P_{1,2}^{\prime}$ & $P_{2,3}$ \\
\hline
\end{tabular}

The opposite pattern for injection and measurement using the classic FIM approach has been applied in previous studies $[14,15]$. Herein, we used a contrasting pattern for FIM, with adjacent patterns for injection and measurement. For this purpose, the electrodes were divided into externals $\left(E_{0}, E_{1}, E_{2}\right.$, and $\left.E_{3}\right)$ and internals $\left(E_{4}, E_{5}, E_{6}\right.$, and $E_{7}$ ) according to the configuration presented in Figure 2. In Table 1, we show the injection and measurement electrodes used for the two proposed FIM approaches.

In the first proposed FIM approach, FIM-I (I, internal), the variable $P$ of equation 3 was equal to $P_{\text {int }}$, which is calculated as $P_{\text {int }}=P_{4,5}+P_{5,6}+P_{5,6}^{\prime}+P_{6,7}$. In the second proposed approach, FIM-IE (IE, Internal-External), $P=P_{\text {int }}+P_{\text {ext }}$ with $P_{\text {ext }}=P_{0,1}+P_{1,2}+P_{1,2}^{\prime}+P_{2,3}$ and $P_{\text {int }}$ as for FIM-I. In the previously described FIM-4 approach [25], variable $P=P_{4,7}+P_{6,7}$. As indicated in Table 1, FIM-I requires two injections of current and four measures of voltage, whereas FIM-IE requires four injections of current and eight measures of voltage. In contrast, FIM-4 requires two injections of current and two measures of voltage. $P_{r e f}$ for each approach is defined as $P$, but considering the saline solution inside the cylindrical cavity equal to that of the phantom $(0.217 \mathrm{~S} / \mathrm{m})$. The FIM approach requires to place the electrodes in a plane in front of the object under study. In the current experiments, this plane corresponds to the lower pelvis and is represented by the underside of the cubic container.

\subsection{Global impedance}

The GI approach is based on reconstructed images of differential EIT (dEIT), in which homogeneous $\left(v_{h}\right)$ and non-homogeneous $\left(v_{n h}^{f}\right)$ vector measurements are taken. The superscript $f$, which ranges from 1 to $N_{f}$, indicates the frame. $N$ is the number of voltage measures per frame and is hence the number of elements of vectors $v_{h}$ and $v_{n h}^{f}$. Differences between $v_{h}$ and $v_{n h}^{f}$ are used in an EIT reconstruction algorithm to calculate changes of conductivity inside the object under study $[11,8,12]$. Hence, variations in potential for any frame $f$ are calculated using the following operation:

$$
\Delta v^{f}(k)=\frac{v_{n h}^{f}(k)-v_{h}(k)}{v_{h}(k)}, \quad k=1, \ldots \mathrm{N}
$$

where $\Delta v^{f}(k), v_{n h}^{f}(k)$ and $v_{h}(k)$ are the $\mathrm{k}$-th elements of the vectors $\Delta v^{f}, v_{n h}^{f}$, and $v_{h}$, respectively. To solve the pixel conductivity vector $I^{f}$, the matrix $R^{f}$ must be 
calculated using a differential EIT reconstruction algorithm. $R^{f}$ has $M$ rows and $N$ columns $\left(M\right.$, number of pixels of the conductivity image). Hence, the vectors $I^{f}$ and $\Delta v^{f}$ are related by equation 5 as follows:

$$
I_{f}=R^{f} \Delta v^{f}
$$

GI of dimensionless units is calculated by adding the values of all pixels of $I^{f}$ for each frame as follows (equation 6):

$$
G I=\sum_{f=1}^{N_{f}} \sum_{k=1}^{N} I^{f}(k)
$$

The Gauss-Newton algorithm was used for image reconstruction and the total variation approach was used for regularization. These algorithms are available in the Electrical Impedance Tomography and Diffuse Optical Tomography Reconstruction Software (EIDORS) [26, 27]. Because the EIT system has 8 electrodes and because voltages at the injection electrode and at its two nearest neighbors are not measured, $N=40$, corresponding with 8 current injections and 5 voltage measurements for each frame. The reconstruction matrix is calculated from a 2D EIT image of 32 by 32 pixels. Therefore, $R^{f}$ has $1024\left(32^{2}\right)$ rows and 40 columns and was solved using a cubic finite element model of 37412 elements implemented in EIDORS.

\section{Results}

In this study, we used three phantoms with cavity radii of $2.50,4.15$ and $5.20 \mathrm{~cm}$, and saline solution conductivity levels of $1.207,1.877$ and $2.610 \mathrm{~S} / \mathrm{m}$. Hence, the total number of experimental configurations for each method (FIM-I, FIM-IE, FIM-4 and $\mathrm{GI}$ ) is nine. Output variables for each method (electrical potential for FIM-I, FIM-IE and FIM-4, and global impedance for GI) were calculated 10 times each, using voltage measures taken 16 seconds apart, but obtained under identical experimental conditions (same size of cylindrical cavity and same conductivity for the saline solution that fills the cavity). For homogeneous measures, we used a reference conductivity of $0.217 \mathrm{~S} / \mathrm{m}$, which corresponds with the conductivity of the bladder wall.

For each of the four methods compared in this study, medians and interquartile ranges of all combinations of cavities and conductivities are presented in Tables 2, 3, 4 and 5. The right-most column of these tables contain $p$-values from Kruskal-Wallis tests for differences between median output variables for each method with varied radii of the cavity and constant conductivity. Differences between output variables were considered insignificant when $p>0.05$, which indicated failure to distinguish between volumes at the given conductivity level. Under these conditions $p$-values are listed in the fourth column. When $p$-values from Kruskal-Wallis tests are $\leq 0.05$, then at least one of the cavities is giving measurements that differ from the other two. Under these conditions, all three possible comparisons of cavities must be made, and the number in the fourth column represents the comparison with the highest $p$-value. 
In Tables 2, 3, 4 and 5 we present $p$-values from Kruskal-Wallis tests for differences between measurements made at constant volumes, for which insignificant differences in conductivity $(p \leq 0.05)$ were associated, indicating robustness of the method against changes of conductivity. In these experiments, we used Kruskal-Wallis tests instead of a one-way ANOVA because not all data sets were normally distributed. The normality was evaluated by applying the Jarque-Bera test to each 36 data sets (3 cavities x 3 conductivities x 4 methods). Each data set consisted of 10 samples of the output variable of one of the four methods included in this paper. For some of these data sets, the pvalues were less than 0.001 , indicating no normality. The present bioimpedance methods were considered suitable for bladder volume estimations if they indicate significant differences between test cavities but no differences relating to conductivity, as follows:

- Table column condition: All values in the last column must be $\leq 0.05$, indicating significant differences between the three test cavities.

- Table row condition: All the values in the last row must be $>0.05$ indicating insignificant differences between measurements of volume despite differing conductivities.

In evaluations using the FIM-I method, electrical potential varies with increasing conductivity and constant volume (Figure 3). Additionally, at a conductivity value of $1.877 \mathrm{~S} / \mathrm{m}, \Delta P$ returned similar values for middle-sized and large cavities $(p=0.3245)$. In addition, FIM-I was sensitive to conductivity.

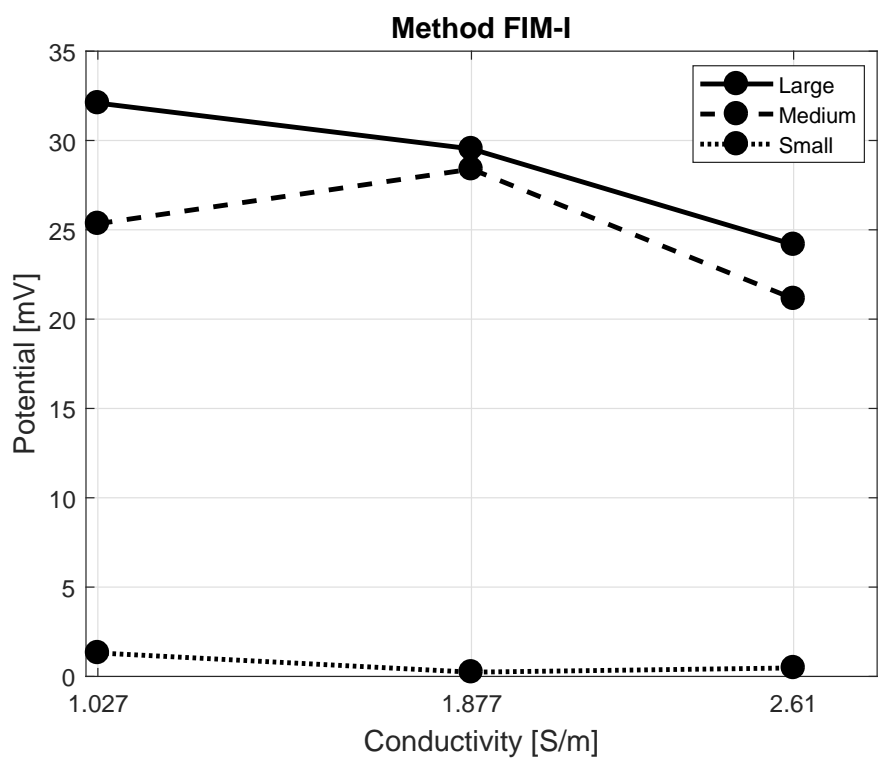

Figure 3. Electrical potential estimated using the FIM-I approach; solid, dashed and dotted lines represent cavities with large, medium and small radii, respectively.

As shown in Figure 4, $\Delta P$ values were approximately constant for each cavity when determined using the FIM-IE method, despite the differing conductivity levels of the saline solution. The values in the last row of Table 3 indicate no significant differences 
Table 2. Median \pm interquartile range for the electrical potential $(\mathrm{mV})$ estimated by the FIM-I approach; p-values were calculated using Kruskal-Wallis tests for differences between median values.

\begin{tabular}{|c|c|c|c|c|c|}
\cline { 3 - 5 } \multicolumn{2}{c|}{} & \multicolumn{3}{c|}{ Radius of the cavity } & \multirow{2}{*}{} \\
\cline { 3 - 5 } \multicolumn{2}{c|}{} & Small & Medium & Large & p-value \\
\hline \multirow{2}{*}{$\approx$} & $\mathbf{1 . 0 2 7}$ & $1.3 \pm 1.0$ & $25.3 \pm 2.5$ & $32.1 \pm 3.7$ & 0.0291 \\
\cline { 2 - 5 } & $\mathbf{1 . 8 7 7}$ & $0.2 \pm 0.1$ & $28.4 \pm 5.5$ & $29.5 \pm 4.8$ & 0.3245 \\
\cline { 2 - 5 } & $\mathbf{2 . 6 1 0}$ & $0.5 \pm 0.5$ & $21.1 \pm 0.8$ & $24.2 \pm 3.0$ & 0.0294 \\
\hline \multirow{3}{*}{} & p-value & 0.0085 & $\leq 0.001$ & $\leq 0.001$ & \multicolumn{2}{c}{} \\
\cline { 2 - 5 } & & &
\end{tabular}

Table 3. Median \pm interquartile range for the electrical potential $(\mathrm{mV})$ estimated by the FIM-IE approach; p-values were calculated using Kruskal-Wallis tests for differences between median values.

\begin{tabular}{|c|c|c|c|c|c|}
\hline & \multicolumn{3}{|c|}{ Radius of the cavity } & \multirow[b]{2}{*}{ p-value } \\
\hline & & Small & Medium & Large & \\
\hline \multirow{4}{*}{$\frac{\xi}{\omega}$} & 1.027 & $0.5 \pm 0.7$ & $33.5 \pm 2.9$ & $42.1 \pm 2.6$ & 0.0298 \\
\hline & 1.877 & $0.2 \pm 0.1$ & $35.7 \pm 4.6$ & $44.5 \pm 5.8$ & 0.0298 \\
\hline & 2.610 & $0.3 \pm 0.1$ & $32.9 \pm 1.8$ & $41.7 \pm 3.4$ & 0.0298 \\
\hline & p-value & 0.3320 & 0.1409 & 0.1959 & \\
\hline
\end{tabular}

$(p>0.05)$, whereas those in the last column of Table 3 show that FIM-IE measurements differ significantly for the three cavities.

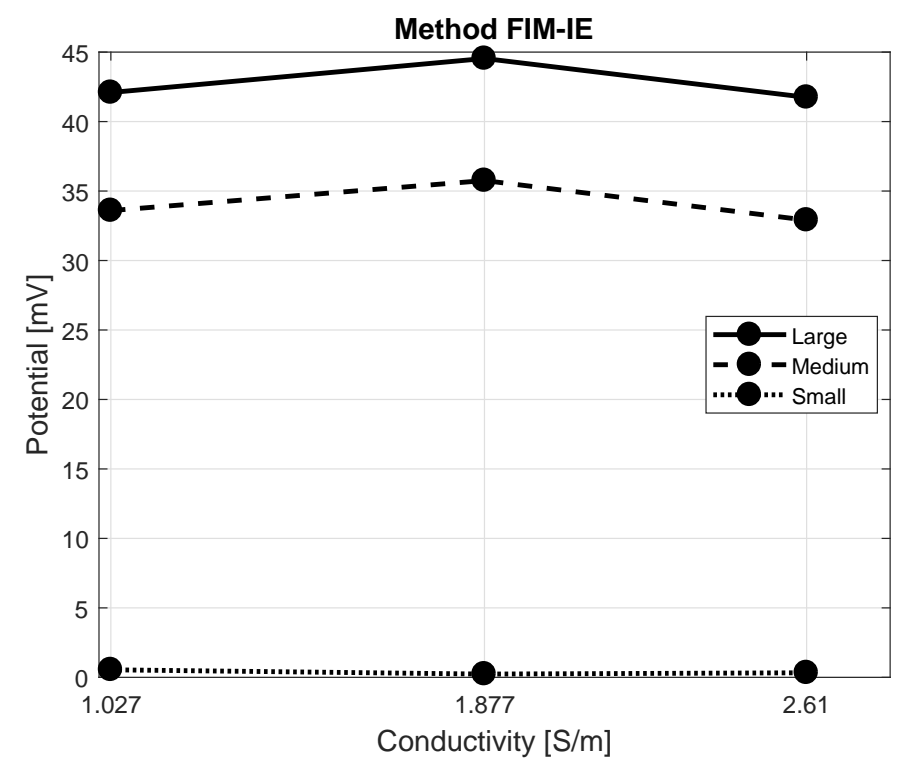

Figure 4. Electrical potential estimated by the FIM-IE approach; solid, dashed and dotted lines represent cavities with large, medium, and small radii, respectively.

As shown in Figure 5, electrical potential from the FIM-4 method varies with increasing conductivity and when cavity volumes are constant. Additionally, at a conductivity of $2.610 \mathrm{~S} / \mathrm{m}, \Delta P$ was similar for middle and large cavities. In all cases FIM-4 failed to differentiate between volumes of at least two cavities (Table 4, last 


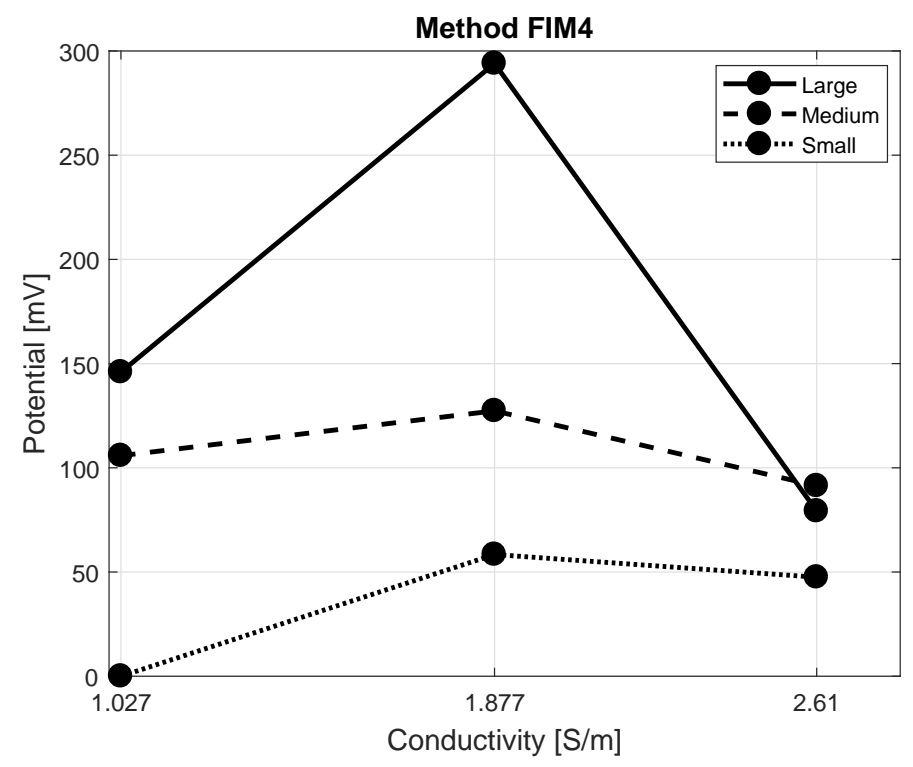

Figure 5. Electrical potential estimated by the FIM-4 approach; solid, dashed and dotted lines represent cavities with large, medium, and small radii, respectively.

Table 4. Median \pm interquartile range for the electrical potential $(\mathrm{mV})$ estimated by the FIM4 approach; p-values were calculated using Kruskal-Wallis tests for differences between median values.

\begin{tabular}{|c|c|c|c|c|c|}
\hline \multirow{2}{*}{\multicolumn{2}{|c|}{ (n) }} & \multicolumn{3}{|c|}{ Radius of the cavity } & \multirow[b]{2}{*}{ p-value } \\
\hline & & Small & Medium & Large & \\
\hline \multirow{3}{*}{$\frac{\Xi}{\mathscr{\Omega}}$} & 1.027 & $0.0 \pm 6.2$ & $105.8 \pm 6.2$ & $145.9 \pm 24.7$ & 0.2751 \\
\hline & 1.877 & $58.4 \pm 19.1$ & $127.3 \pm 154.9$ & $294.0 \pm 55.6$ & 0.1825 \\
\hline & 2.610 & $47.5 \pm 16.1$ & $91.3 \pm 40.3$ & $79.2 \pm 59.6$ & 0.4254 \\
\hline & p-value & $\leq 0.001$ & 0.0012 & $\leq 0.001$ & \\
\hline
\end{tabular}

Table 5. Median \pm interquartile range for the dimensionless values given by the GI approach; differences between median values were identified using Kruskal-Wallis tests.

\begin{tabular}{|c|c|c|c|c|c|}
\hline & \multicolumn{3}{|c|}{ Radius of the cavity } & \multirow[b]{2}{*}{ p-value } \\
\hline & & Small & Medium & Large & \\
\hline \multirow{4}{*}{$\frac{g}{\text { च }}$} & 1.027 & $78.7 \pm 93.2$ & $1362.2 \pm 112.5$ & $1018.2 \pm 725.5$ & 0.6300 \\
\hline & 1.877 & $54.1 \pm 82.1$ & $2537.5 \pm 103.4$ & $3751.8 \pm 462.0$ & 0.0292 \\
\hline & 2.610 & $259.7 \pm 32.8$ & $3484.7 \pm 26.3$ & $4955.5 \pm 128.9$ & 0.0296 \\
\hline & p-value & 0.0012 & $\leq 0.001$ & $\leq 0.001$ & \\
\hline
\end{tabular}

column). Like FIM-I, measurements of electric potential from the FIM-4 approach were largely dependent on conductivity (Table 4).

In assessments using GI, median values varied with increasing conductivity of saline solutions (Figure 6) when cavity volumes were kept constant. In addition, at a conductivity of $1.027 \mathrm{~S} / \mathrm{m}$, GI values for the large cavity were less than those for the middle cavity. Yet for a conductivity of $1.877 \mathrm{~S} / \mathrm{m}$, the GI values for the large cavity were greater than for the middle-sized cavity. As listed in Table 5, GI fails in the row condition for all three cavity sizes, and fails the column condition for the lowest cavity size. 


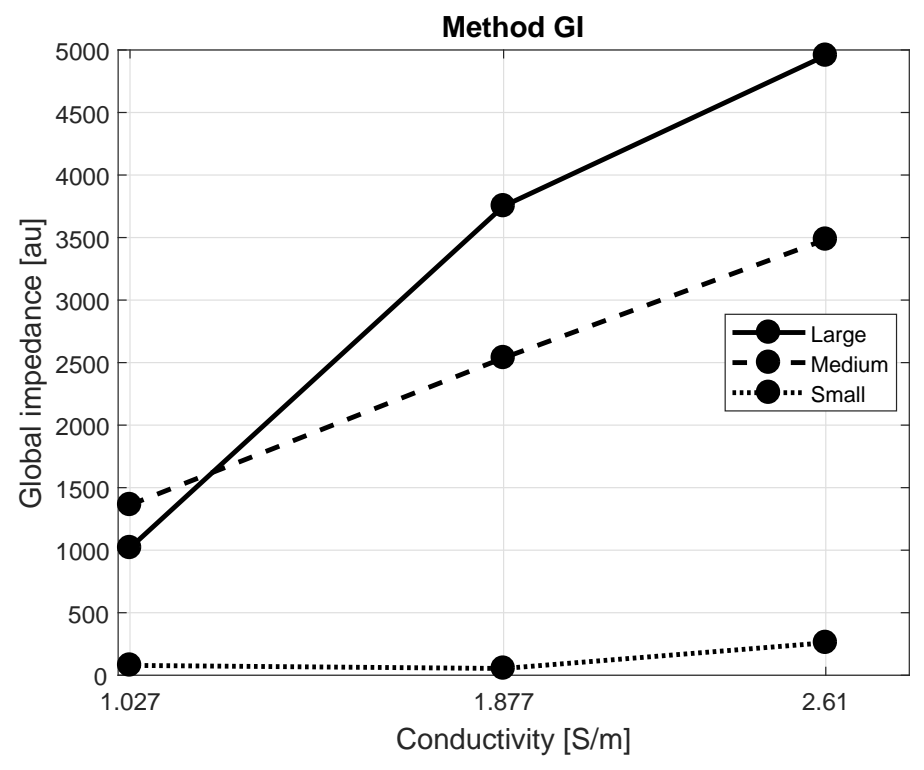

Figure 6. Global impedance estimated by the GI approach; solid, dashed and dotted lines represent cavities with large, medium, and small radii, respectively.

In summary, FIM-I, FIM4, and GI approaches do not satisfy the row condition of similar values over a range of conductivities. Specifically, all three methods are subject to electrical conductivity of saline solutions. These three methods also fail the column condition of differing measurements between different sized cavities for at least one of the cavities. In contrast, the FIM-IE approach satisfies both conditions and returns electrical potential values that vary only with cavity sizes.

\section{Discussion}

A key drawback of FIM and GI approaches to measuring volumes is that the measurements (electrical potential for FIM-I, FIM-IE and FIM-4, and global impedance for GI) are highly subject to conductivity, which varies in urine according to diet and various other physiological conditions. Hence, fundamental to deciding whether a bioimpedance approach can be used to inform bladder evacuation in people with UI is whether the method estimates the volume of an object with unknown conductivity. To address this limitation, we compared GI with three FIM approaches. Specifically, we determined whether each approach differentiates between three cavities of different volumes of liquid with unknown conductivity.

GI has been recently proposed to estimate bladder volumes, predominantly because a linear relationship with volumes of objects has been shown. In a previous study [28] of nine male paraplegic patients, a strong Pearson's correlation was reported between GI and bladder volume. This correlation was negative for four patients because the conductivity of urine is higher than that of surrounding organs. Yet, positive correlations were identified in other five patients, and these were attributed to bladder movements outside measurement areas. In all of the present experiments with GI (Figure 6), these 
correlations were negative because the conductivities of the saline solutions $(1.027,1.877$, and $2.610 \mathrm{~S} / \mathrm{m})$ were higher than that of the background $(0.217 \mathrm{~S} / \mathrm{m})$. In the previous study [28], conductivity was kept constant in urine samples by filling bladders with a contrast fluid of known conductivity. As a consequence, the effects of electrical properties of urine were not investigated in their study. Additionally, the effects of patient urine production were reduced by performing the experiment over only $30 \mathrm{~min}$.

In a simulation study comparing errors of volume estimates, noise tolerance, and sensitivity to urine conductivity of GI with those of three EIT-based methods [8], average errors were lower than $5 \%$ and had insignificant standard deviations after calibrating the equation relating volume with GI. But changes in urine conductivity required further calibration to avoid over or under-estimation of volumes. In another study, GI was compared with other bioimpedance approaches [12], and GI values largely depended on the conductivity of urine. The data presented herein (Table 5) show that for a constant fluid volume significant differences $(p<0.05)$ in GI values follow conductivity changes. These results are in agreement with previous studies [8], [12], indicating that GI can be used to estimate volumes only when conductivity of the object under study remains constant.

The FIM-4 approach was proposed in a previous study [29], and comprises two mutually orthogonal tetrapolar measurements to estimate the impedance of an object that is located below the plane formed by the electrodes. Based on FIM-4, a method for organ volume estimation was proposed [16], and it was shown that simulations that are based on finite elements give linear relationships between volumes of study objects and sensitivity, which is defined as the quotient of impedance variation and the distance between electrodes. In addition, simulation experiments that were performed in a saline tank demonstrated that the constant that relates volume and sensitivity depends on the conductivity of the object and on its depth with respect to the plane of electrodes. Similarly, we show that for a constant volume, the values given by FIM-I and FIM-4 approaches depend on the conductivity of the saline solution (Tables 2 and 4). Our experiments with FIM-IE, however, showed no dependence of volume measurements on the conductivity of saline solutions (Table 3), and strict dependence on the volume of the cavity. The main difference between these three FIM approaches is the additional information required to calculate variations of electrical potential. Unlike FIM-I and FIM-4 procedures, FIM-IE is performed by injecting current and measuring voltage on internal and external electrodes. When current is injected through internal electrodes, voltage is measured at the external electrodes, and vice versa.

Each of the present bioimpedance approaches for estimating bladder volumes requires a calibration procedure that begins by emptying the patient's bladder and then using urodynamics to relate bladder volumes to the bioimpedance variable. The resulting equations relate measurements with volumes but are only valid when urine conductivity is equal to that during the calibration phase. Because urine conductivity varies physiologically, it is important to use bioimpedance approaches for which measured variables are independent of urine conductivity. Herein, we demonstrate that 
the FIM-IE approach meets these criteria, although more tests are required. Initially, such tests will require a phantom bladder with irregularly shaped cavities of variable volume that allow experiments with more than three volumes. Additionally, to make the phantom prototype more realistic, the container will be cylindric and the electrodes will be placed on its front side. Another aspect to consider in future experiments is that the conductivity of urine in the bladder is not constant as its volume increases. The ensuing experiments will verify the generalizability of the FIM-IE approach.

\section{Acknowledgments}

This work was funded by the InnovAccion-Cauca project, the research group of Automation of Universidad del Cauca (501100005682), and by the internal resources of the group of Electronic and Biomedical Instrumentation of the Technical University of Catalonia.

\section{References}

[1] Ha Bum Jung, Hyung Jee Kim, and Sung Tae Cho. A current perspective on geriatric lower urinary tract dysfunction. Korean journal of urology, 56(4):266-275, 2015.

[2] Lauren N. Wood and Jennifer T. Anger. Urinary incontinence in women. BMJ, 349:g4531, 2014.

[3] Waleed Al Taweel and Raouf Seyam. Neurogenic bladder in spinal cord injury patients. Research and reports in urology, 7:85, 2015.

[4] Naoki Yoshimura and Michael B Chancellor. Differential diagnosis and treatment of impaired bladder emptying. Reviews in urology, 6(Suppl 1):S24, 2004.

[5] Jerome Bickenbach, Alana Officer, Tom Shakespeare, Per von Groote, et al. Lesiones de la médula espinal: perspectivas internacionales, resumen. Technical report, Swiss Paraplegic Research, 2014.

[6] Stefania Musco, Barbara Padilla-Fernández, Giulio Del Popolo, Matteo Bonifazi, Bertil F. M. Blok, Jan Groen, Lisette 't Hoen, Jürgen Pannek, Jerome Bonzon, Thomas M. Kessler, Marc P. Schneider, Tobias Gross, Gilles Karsenty, Véronique Phé, Rizwan Hamid, Hazel Ecclestone, and David Castro-Diaz. Value of urodynamic findings in predicting upper urinary tract damage in neuro-urological patients: A systematic review. Neurourology and Urodynamics, 37(5):1522$1540,2018$.

[7] Seong Ho Lee, Sung Tae Cho, Hae Ri Na, Seok Bum Ko, and Moon Ho Park. Urinary incontinence in patients with a lzheimer's disease: Relationship between symptom status and urodynamic diagnoses. International Journal of Urology, 21(7):683-687, 2014.

[8] Thomas Schlebusch, Steffen Nienke, S Leonhardt, and M Walter. Bladder volume estimation from electrical impedance tomography. Physiological measurement, 35(9):1813, 2014.

[9] Marjoyre Anne Lindozo Lopes and Elenice Dias Ribeiro de Paula Lima. Continuous use of intermittent bladder catheterization-can social support contribute? Revista latino-americana de enfermagem, 22(3):461-466, 2014.

[10] Yaning Li, Yinglin Peng, Xin Yang, Shipei Lu, Jinwu Gao, Chengguang Lin, and Rihui Li. Analysis of measurement electrode location in bladder urine monitoring using electrical impedance. Biomedical engineering online, 18(1):34, 2019.

[11] Steffen Leonhardt, Axel Cordes, Harry Plewa, Robert Pikkemaat, Irina Soljanik, Klaus Moehring, Hans J Gerner, and Rüdiger Rupp. Electric impedance tomography for monitoring volume and size of the urinary bladder. Biomedizinische Technik/Biomedical Engineering, 56(6):301-307, 2011. 
[12] Thomas Schlebusch, Jakob Orschulik, Jaakko Malmivuo, Steffen Leonhardt, Dorothea Leonhäuser, Joachim Grosse, Michael Kowollik, Ruth Kirschner-Hermanns, and Marian Walter. Impedance ratio method for urine conductivity-invariant estimation of bladder volume. Journal of Electrical Bioimpedance, 5(1):48-54, 2019.

[13] Rihui Li, Jinwu Gao, Yaning Li, Junpeng Wu, Zhanqi Zhao, and Yang Liu. Preliminary study of assessing bladder urinary volume using electrical impedance tomography. Journal of Medical and Biological Engineering, 36(1):71-79, 2016.

[14] K.S. Rabbani, M Sarker, M.H.R Akond, and T. Akter. Focused impedance measurement (FIM): a new technique with improved zone localization. Annals of the New York Academy of Sciences, 873(1):408-420, 1999.

[15] KS Rabbani and MAS Karal. A new four-electrode focused impedance measurement (fim) system for physiological study. Annals of biomedical engineering, 36(6):1072-1077, 2008.

[16] Sayed Parvez Ahmed, M Abdul Kadir, Rubina Rahman, Golam Dastegir Al Quaderi, and K Siddique-e Rabbani. Determination of organ volume using focused impedance method (fim): A simulation approach. Bangladesh Journal of Medical Physics, 7, 2015.

[17] Khondkar Siddique-e Rabbani. Focused impedance method: Basics and applications. In Bioimpedance in Biomedical Applications and Research, pages 137-185. Springer, 2018.

[18] Masum Iquebal and K Siddique-e Rabbani. Correlation of liquid volume in stomach to electrical transfer impedance measurements using fim. Bangladesh Journal of Medical Physics, 6(1), 2014.

[19] M Abdul Kadir, Tanvir Noor Baig, and K Siddique-e Rabbani. Focused impedance method to detect localized lung ventilation disorders in combination with conventional spirometry. Biomedical Engineering: Applications, Basis and Communications, 27(03):1550029, 2015.

[20] Eoghan Dunne, Brian McGinley, Martin O'Halloran, and Emily Porter. A realistic pelvic phantom for electrical impedance measurement. Physiological measurement, 39(3):034001, 2018.

[21] PA Hasgall, F Di Gennaro, C Baumgartner, E Neufeld, B Lloyd, MC Gosselin, D Payne, A Klingenböck, and Kuster N. It'is database for thermal and electromagnetic parameters of biological tissues, 2018.

[22] Víctor Hugo Mosquera, Adrian Arregui, Ramon Bragós Bardia, and Carlos Felipe Rengifo. Implementation of a low cost prototype for electrical impedance tomography based on the integrated circuit for body composition measurement afe4300. In Proceedings of the 11th International Joint Conference on Biomedical Engineering Systems and Technologies (BIOSTEC 2018): January 19-21, 2018: Funchal, Madeira, Portugal, pages 121-127. Scitepress, 2018.

[23] Van Yang, P Aroul, and K Wen. Impedance measurement with the afe4300. Application Repor. SBAA202-October, 2013.

[24] Samiron Kumar Saha, Golam Dastegir Al-Quaderi, and Khondkar Siddique-e Rabbani. 3d sensitivity of 8-electrode FIM through experimental study in a phantom. Bangladesh Journal of Medical Physics, 6(1), 2014.

[25] Naimul Islam, K Siddique-e Rabbani, and Adrian Wilson. The sensitivity of focused electrical impedance measurements. Physiological measurement, 31(8):S97, 2010.

[26] Sanwar Ahmad, Thilo Strauss, Shyla Kupis, and Taufiquar Khan. Comparison of statistical inversion with iteratively regularized gauss newton method for image reconstruction in electrical impedance tomography. Applied Mathematics and Computation, 358:436-448, 2019.

[27] Vernoon Ang, MHF Rahiman, and RA Rahim. Study of gauss-newton and total variation image reconstruction approach in imaging the phantom in concrete using electrical resistance tomography. In Symposium on Intelligent Manufacturing and Mechatronics, pages 487-496. Springer, 2019.

[28] Leonhardt Steffen, Cordes Axel, Plewa Harry, Pikkemaat Robert, Soljanik Irina, Moehring Klaus, J. Gerner Hans, and Rupp Rüdiger. Electric impedance tomography for monitoring volume and size of the urinary bladder. Biomedizinische Technik/Biomedical Engineering, 56(6):301, 2011.

[29] K. S. Rabbani and M. A. S. Karal. A new four-electrode focused impedance measurement (FIM) system for physiological study. Annals of Biomedical Engineering, 36(6):1072-1077, Jun 2008. 